\title{
Prediction of mechanical properties of nanocrystalline materials using Voronoi FE models of representative volume elements
}

\author{
Panagiotis Bazios ${ }^{1}$, Konstantinos Tserpes ${ }^{1, *}$, and Spiros Pantelakis ${ }^{1}$ \\ ${ }^{1}$ Laboratory of Technology and Strength of Materials, Department of Mechanical Engineering and \\ Aeronautics, University of Patras, 26500 Rion, Greece
}

\begin{abstract}
In the present work, a numerical model is developed to predict the mechanical properties of nanocrystalline materials using a Finite Element Analysis. The model is based on Representative Volume Elements (RVE) in which the microstructure of the material is described using the Voronoi tessellation algorithm. The use of the Voronoi particles was based on the observation of the morphology of nanocrystalline materials by Scanning Electron and Transmission Electron Microscopy. In each RVE, three-dimensional modelling of the grain and grain boundaries as randomlyshaped sub-volumes is performed. The developed model has been applied to pure nanocrystallline copper taking into account the parameters of grain size and grain boundary thickness. The mechanical properties of nanocrystalline copper have been computed by loading the RVE in tension. The numerical results gave a clear evidence of grain size effect and the Hall-Petch relationship, which is a consequence of macroscopic strain being preferentially accumulated at grain boundaries. On the other hand, for a given grain volume fraction, the results for elastic moduli showed no effect of the grain size. The model predictions have been validated successfully against numerical results from the literature and predictions of the Rule of Mixtures and the Mori-Tanaka analytical model.
\end{abstract}

\section{Introduction}

Nanocrystalline (NC) metals have pulled a considerable interest in the course of the last two decades because of their exceptional mechanical properties [1]. One of the features of these materials, which is best examined and described, is their mechanical behaviour [2]. It is commonly known that contrasted to their microcrystalline materials, nanomaterials are characterized by higher values of the yield stress, tensile strength, and hardness, whereas their plasticity and fracture toughness are lower [3]. Another essential parameters which depict the mechanical behaviour of materials are their modulus of elasticity $-E$ and Shear Modulus - G. In the case of nanomaterials there are however some problems with determining the elastic moduli. The conceivable connection between the estimation of this modulus and the structure of nanomaterials is hard to depict. This is so since these materials are manufactured by different methods, various types of samples are prepared for their examinations, and various methods are employed for measuring their properties.

However, whichever is the production technique, nanocrystalline materials undergo grain coarsening which is a thermodynamic driven process. Due to the monotonic reduction of the Gibbs free energy versus the grain size, nanocrystalline materials exhibit advanced mechanical behaviour, which, however, is prone to thermodynamic instability at elevated

*Corresponding author: kitserpes@upatras.gr 
temperatures. The most promising production technique is the High Energy Ball Milling in which the grain size decrease with the milling time down to a constant value which varies with the melting temperature of the material. This phenomenon implies a balance between defect creation and recovery during deformation. Provided the problems of contamination during the milling process (by attritors or absence of inert gas environment) and powder consolidation without coarsening are solved, mechanical attrition could be used for producing nanocrystalline materials in notable quantities.

From the above, it becomes evident that experimental characterization of the mechanical behaviour of nanocrystalline materials is a very difficult task due to the inability of the research community to produce enough material for test specimens. On the other hand, these technological restrictions have been the impetus for the development of numerical models capable of correlating the material structure (grain size, volume fraction of each phase, etc) with the mechanical properties of nanocrystalline materials.

In the present work, a numerical model is created to predict the mechanical properties of nanocrystalline materials utilizing a Finite Element Analysis. The model is based on Representative Volume Elements (RVE) in which the microstructure of the material is described using the Voronoi tessellation algorithm and its results are in consistence with several scientific publications.

\section{Analytical approach}

The following model is presented in its general formulation. The essential idea (Fig. 1) of the model assumes that $\mathrm{NC}$ material is a composite containing of two discrete phases: the grain core (inclusions) and the grain boundaries (matrix). Additionally, a perfect bonding between the two phases is assumed. To simplify the analysis, grain boundary triple junctions are incorporated in the grain boundaries in terms of volume. This simplification should not have a major influence on the results for materials with a grain size greater than $10 \mathrm{~nm}$ since the volume.

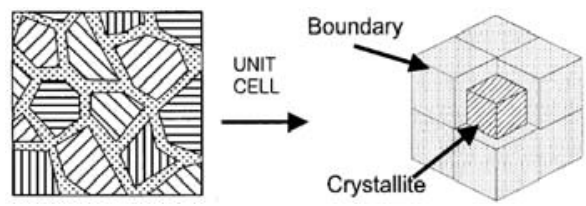

Fig. 1. Illustration of Rule of Mixture approach

Nanocrystalline materials generally refer to the class of materials whose average grain size is below $100 \mathrm{~nm}$. As many atoms reside in the grain boundary regions in this case the volume fraction of the grain-boundary phase is not zero. Regarding the grain size (diameter) $\mathrm{d}$ and grain-boundary thickness $\mathrm{t}$, the volume fraction of the grains can be approximated by

$$
V F_{c r}=\frac{d^{3}}{(d+t)^{3}}
$$

and that of the grain-boundary phase by $V F_{g b}=1-V F_{c r}$. Moreover, the inclusion phase (crystallite) will also be considered homogeneous. In a coarse-grained material we have $t / d \rightarrow 0$; the whole polycrystal is then fully occupied by the grains and its elastic behaviour is simply the averaged behaviour of these crystallites. However, for a nanocrystalline material, for instance at $d=20 \mathrm{~nm}$ and $\mathrm{t}=1 \mathrm{~nm}$, the volume fraction of the grain-boundary phase is about $14 \%$ and its contribution to the overall elastic behaviour presents some fluctuations.

Fig. 1 demonstrates an illustration of the phase mixture model in an NC material. A cubic 
unit cell of the NC material comprises of a grain interior and grain boundaries. It was indicated [4] that the results of the finite element calculations for the analysis of plastic deformation in $\mathrm{NC}$ materials are almost the same as those obtained using the rule of mixtures. Therefore, in this study, the following simple rule of mixtures based on the volume fractions of the components is used for the analysis of deformation behaviour:

$$
E=V F_{c r} E_{c r}+V F_{g b} E_{g b}
$$

where the subscripts cr and gb refer to crystallite and grain boundary, respectively. The stress in each of the two components of the 'composite' is calculated using the assumption that the strains in both phases are the same and are equal to the macroscopic applied strain.

\section{Numerical approach}

Atomistic simulation methods provide exceptional insight into the structural behaviour of $\mathrm{NC}$ materials. However, their exploitation for predicting the respective mechanical properties is very difficult due to inherent restrictions of the methods. In this manner, a numerical model of NC materials aims to provide a tool for the design-by-analysis of the essential NC material microstructural features in order to obtain the desired mechanical behaviour.

The proposed approach relies on the development of Representative Volume Elements (RVE) of the NC material. Owing to the small size of RVEs (in the case of NC materials they are about $100 \mathrm{~nm}$ edge cubes) detailed morphology of materials microstructures using different types of algorithms can be modeled. Observation of NC materials SEM/TEM [5] images (Fig. 2) from several publications revealed that $\mathrm{NC}$ materials consist of randomly polyhedral shaped grains. In terms of the realistic microstructure of NC materials into RVE, the micro-structure geometry has been developed using Voronoi tessellation algorithm. In each RVE, detailed three-dimensional modelling of the grain and grain boundaries as randomly-shaped sub-volumes is performed (Fig. 3). As considered volume fraction of grains and grain boundaries play a significant role on the overall calculated mechanical behaviour, these volume fractions can be parametrically defined in the NC model.

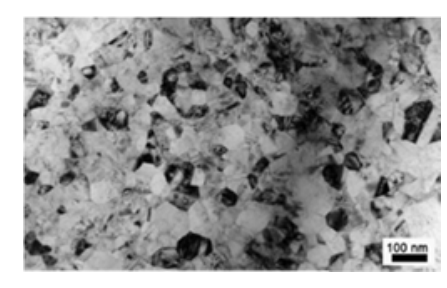

Fig. 2. A TEM bright-field image of the NC Copper [5]

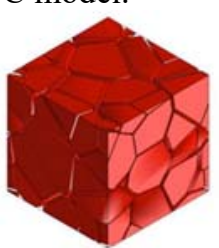

Fig. 3. Unmeshed Voronoi Tesselation

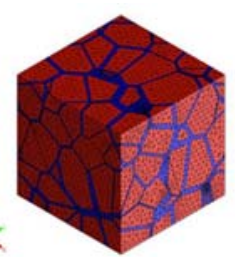

Fig. 4. Representative RVE of a NC material

The RVE geometrical model is meshed utilizing tetrahedral finite elements (Fig. 4), proper material laws at each sub-volume are assigned.

The size of individual grains was described by the equivalent diameter, $d$, defined as the diameter of a circle with an area equal to the area of a given grain. The model structures were characterized by the average grain diameter, D, which ranged from 10 to $100 \mathrm{~nm}$.

In order to reflect a significant fraction of atoms residing at the grain boundaries in 
nanograined metals, it was assumed that grain boundaries have a non-zero thickness of $1 \mathrm{~nm}$. This thickness concurs well with estimates provided by ab initio computations [6].

It was assumed that grain boundaries and grain interiors exhibit the elastic-plastic properties described by the following relationships:

$$
\begin{gathered}
\sigma_{c r / g b}=E_{c r / g b} \varepsilon \text { for } \varepsilon \leq \varepsilon_{0} \\
\sigma_{c r / g b}=E_{c r / g b} \varepsilon_{0}+\theta_{c r / g b}\left(\varepsilon-\varepsilon_{0}\right) \text { for } \varepsilon>\varepsilon_{0}
\end{gathered}
$$

where $\mathrm{e}_{0}$ is the elastic strain at the yield point, $\mathrm{E}$ is the Young's modulus and $\mathrm{h}$ is the workhardening coefficient. It was further assumed that the flow stress of grain interiors is governed by the Hall-Petch relationship:

$$
\sigma_{c r}=\sigma_{0}+K(d)^{-m}
$$

where $d$ is the equivalent diameter of a given grain. It should be noted that $m=1 / 2$ is consistent with the Hall-Petch dependence as such.

The plasticity of grain boundaries was approached based on the Hill criterion [7], which in general accounts for differences in the yield strength in orthogonal directions. The equivalent stress can be described as:

$$
\sigma_{g b}=\left(\frac{1}{2}\{\sigma\}^{T}[M]\{\sigma\}-\frac{1}{3}\{\sigma\}^{T}\{L\}\right)^{\frac{1}{2}}
$$

where $[\mathrm{M}]$ is a matrix of yield stress variations with orientation and $\{\mathrm{L}\}$ describes the difference between tension and compression of yield strengths:

$$
[M]=\left[\begin{array}{cccccc}
M_{11} & M_{12} & M_{13} & 0 & 0 & 0 \\
M_{12} & M_{22} & M_{23} & 0 & 0 & 0 \\
M_{13} & M_{23} & M_{33} & 0 & 0 & 0 \\
0 & 0 & 0 & M_{44} & 0 & 0 \\
0 & 0 & 0 & 0 & M_{55} & 0 \\
0 & 0 & 0 & 0 & 0 & M_{66}
\end{array}\right] \text { where } M_{j j}=\frac{K}{\sigma+j \sigma_{-j}}, j=1 \ldots 6
$$

where $\sigma_{+j}$ and $\sigma_{-j}$ are tensile and compressive yield strengths in the direction $\mathrm{j}, \mathrm{j}=\mathrm{x}, \mathrm{y}, \mathrm{z}$, $\mathrm{xy}, \mathrm{yz}, \mathrm{xz}$. The mechanical parameters assumed in the computations are summarized in Table

\begin{tabular}{|c|c|c|c|c|}
\hline & $\begin{array}{c}\text { Young } \\
\text { modulus } \\
{[\mathrm{GPa}]}\end{array}$ & $\begin{array}{c}\text { Poisson } \\
\text { ratio }\end{array}$ & Yield strength $[\mathrm{MPa}]$ & $\begin{array}{c}\text { Work } \\
\text { hardening } \\
\text { coefficient } \\
{[\mathrm{MPa}]}\end{array}$ \\
\hline $\begin{array}{c}\text { Grain } \\
\text { interiors }\end{array}$ & 120 & 0.336 & $\begin{array}{c}\sigma_{\mathrm{i}}=\sigma_{0}+\mathrm{k}_{\mathrm{y}}(\mathrm{d})^{1 / 2} \\
\sigma_{0}=33[\mathrm{MPa}] \\
\mathrm{k}_{\mathrm{y}}=0.135\left[\mathrm{MPa} \mathrm{m}^{1 / 2}\right]\end{array}$ & 315 \\
\hline $\begin{array}{c}\text { Grain } \\
\text { boundaries }\end{array}$ & 96 & 0.336 & $\begin{array}{c}\sigma_{g b}^{j}=\sigma_{0} \mathrm{Re}^{\mathrm{j}} \text { where } \mathrm{j}=\mathrm{xy}, \mathrm{xy} \\
\mathrm{Re}^{\mathrm{x}}=15, \mathrm{Re}^{\mathrm{y}}=25, \mathrm{Re}^{\mathrm{xy}=15} \\
\sigma_{g b}^{x}, \sigma_{g b}^{y}: \text { yield flow for direction parallel and } \\
\text { perpendicular to the grain boundary } \\
\sigma_{g b}^{x y}: \text { yield flow for shearing of grain boundary }\end{array}$ & 252 \\
\hline
\end{tabular}
1. The value of $\sigma_{0}$ in Eq. (5) was $33 \mathrm{MPa}$ in all cases. The value of $\mathrm{k}$ was $0.135 \mathrm{MPa}^{*} \mathrm{~m}^{1 / 2}$ and the work-hardening coefficient $\theta_{i}$ is equal to $315 \mathrm{MPa}$ from the indicative material parameters of Hollomon's equation. These values are representative for copper [8] and for $\mathrm{d}=10 \mathrm{~nm}$ they result in the same value of macroscopic flow stress.

Table 1. Mechanical properties assumed in the computations. 
The elastic modulus of the grain boundaries, $\mathrm{E}_{\mathrm{gb}}$, was $80 \%$ of the value for the grain interiors, $E_{\mathrm{cr}}$ and the work-hardening coefficient for the grain boundaries, $\theta_{\mathrm{gb}}$, was $5 \%$ of $\theta_{\mathrm{cr}}$. The assumption of the lower value of the grain boundary elastic constant was based on the results of ab initio computations reported in [9] and experimental data published in [10]. The yield flow of the grain boundaries were constant and equal to $500 \mathrm{MPa}$ for shearing and elongation along the grain boundaries plane, and $825 \mathrm{MPa}$ for deformation in the perpendicular direction. These values have been selected in order to model the situation in which the transition from grain boundary strengthening to grain boundary softening is relatively smooth. For lower values of the grain boundary flow stresses, nearly entire plastic deformation is accommodated at the grain boundaries and these stresses determine the macroscopic flow stress of the polycrystalline aggregate [11].

FEM computations were performed utilizing ANSYS software with SOLID 185 elements. Such elements permit non-linear elastic-plastic and anisotropic characteristics and modelling of relatively large deformations. Each phase of the RVE (grain interiors and grain boundaries) have been simulated by using the bilinear isotropic hardening approach (BISO). In the simulations, it was assumed that the modelled structure is subjected to tensile straining.

Moreover, the resulting displacement at the surface of the unit volume has been normalized by its length in the vertical direction to obtain the macroscopic strain, $\mathrm{e}_{\mathrm{m}}$. In an uniaxial tensile test, boundary conditions applied for the microstructure model are shown in Fig. 5.

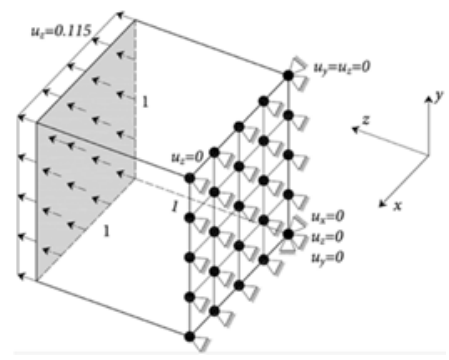

Fig. 5. Applied boundary conditions in the tensile test.

The elastic moduli of the material (Young's Modulus of Elasticity and Shear Modulus) can be numerically predicted without the need to perform an extensive mechanical test campaign. For validation purposes, a limited number of experiments is necessary. The developed methodology will provide the means to design the essential NC material microstructure based on the required material properties.

\section{Results and discussions}

Three-dimensional Voronoi particle RVE models were randomly created utilizing the technique of Christoffersen [12] grain algorithm. Numerical investigations were made so as to determine the influence of the size of the Voronoi particles (grains) on the effective material properties of these composite-like materials ( $\mathrm{NC}$ materials). The results demonstrated that the influence of the size of the Voronoi particles on the effective material properties was not noteworthy in the linear elastic case. On the other hand, the influence of the grain size has been appeared to be prevailing in the non-linear case by showing evidently the importance of the Hall-Petch effect at the nanoscale. Taking this fact into consideration and, using different sizes of the Voronoi particles, the effective material properties of these composites were acquired for up to $95 \%$ volume fractions for the investigation of the elastic moduli. The determination of the yield stress has been made by using a constant volume 
fraction model by varying only the grain size.

The results of the numerical approach were compared with different analytical methods which are Mori-Tanaka estimates (M-T) and Rule of Mixtures Method (ROM) for the elastic case.

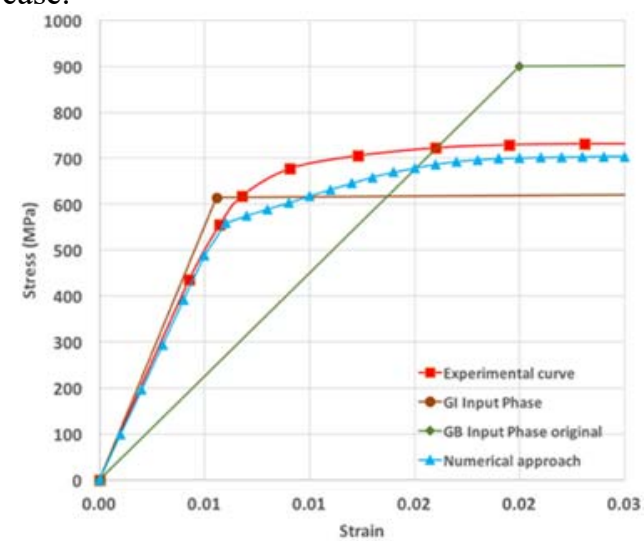

Fig. 6. The computed stress-strain curves for nanocrystals with grain size of 54nm and Young's Moduli for grain interiors and grain boundaries, $110 \mathrm{GPa}$ and $45 \mathrm{GPa}$ respectively [4].

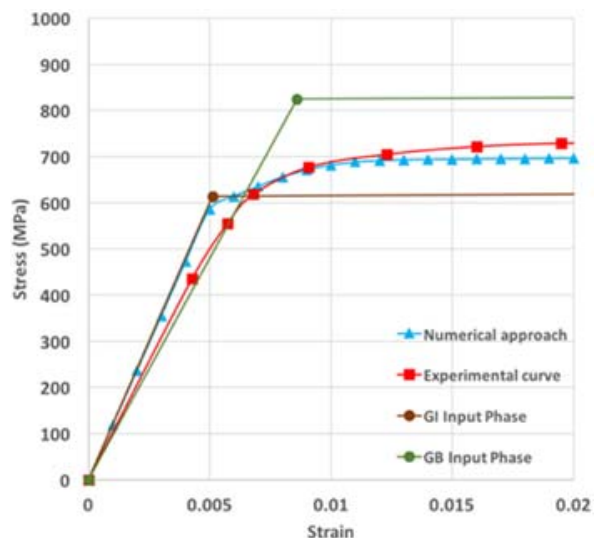

Fig. 7. The computed stress-strain curves for nanocrystals with grain size of $54 \mathrm{~nm}$ and Young's Moduli for grain interiors and grain boundaries, 120GPa and $96 \mathrm{GPa}$ respectively [11].

For validation purposes, the proposed numerical approach has been compared with an experimental result from open literature [13]. The presented numerical approach has been compared with the aforementioned experimental results by varying only the elastic parameters in order to investigate the numerical sensitivity based on input values of one additional numerical approach [10].

In [4], a research project was focused on the numerical investigation of a nanocrystalline copper. The elastic modulus $\mathrm{E}$ of the grain boundary phase could not be calculated from the available experimental data. Grain boundaries had been considered, as a first approximation, to have a random atomic distribution similar to the amorphous structure. For calculation purposes, it was assumed that the grain boundary behaves like an amorphous phase. It is generally accepted that the yield stress of amorphous alloys is between E/50 and $\mathrm{E} / 80$ while $\mathrm{E}$ is $60-75 \%$ that of the corresponding equilibrium crystalline alloy [14].

From Fig. 6, in which RVE consists of elastic moduli of $110 \mathrm{GPa}$ and $45 \mathrm{GPa}$ for grains and grain boundaries respectively, it can be observed that the differences of the numerical effective elastic properties were very small between the numerical approach and the experimental test. On the other hand, the plastic region seems to have a divergence on the yield strength point and the overall plastic curve compared with the experimental plastic region. This may be caused by the bilinear isotropic hardening approach of the elastoplastic behavior of each input numerical phase (grain and grain boundaries), which does not allow to approximate the experimental curve with lower deviation. In case of implementation of a sophisticated multilinear hardening approach, the aforementioned numerical approach may present more efficient results, closer to the experimental stress-strain curve.

In Fig. 7, the data of the Table 1 have been applied on the numerical approach. The numerical stress-strain curve has a deviation from the experimental curve in the elastic region but it approximates adequately the plastic region due to the implementation of lower yield strength of grain boundaries' phase. The definition of the aforementioned yield strength value is based on the plastic anisotropic coefficients [11].

Different studies were made to determine the effect of the size of the Voronoi particles on effective material properties of these NC materials. Fig. 8 explains the effect of the size 
of the Voronoi particles on the effective material properties. Here the thickness of the grain boundary remains constant and by varying the size of the particles, effective material properties were obtained at $83 \%$ - 95\% volume fraction. From Fig. 8 , it can be observed that there were no significant variations by changing the size of the Voronoi particles on the effective material properties.
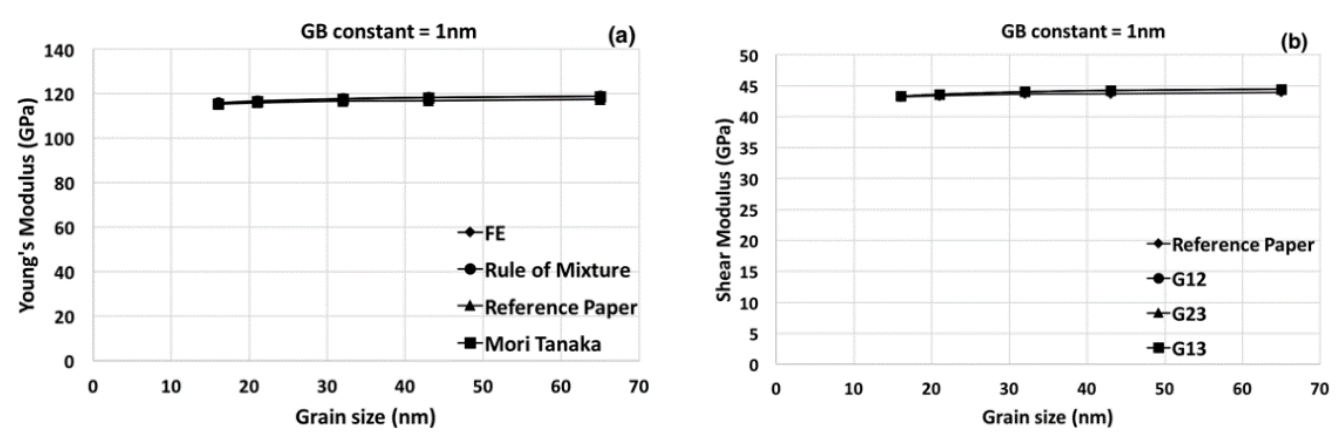

Fig. 8. The numerical and analytical results of nanocrystalline materials for Young's Modulus (a) and Shear Modulus (b) taking the grain boundary thickness as a constant value of $1 \mathrm{~nm}$.

Although slight variations can be observed in the effective material properties with change in the size of the particles, these might be due to greater number of particles inside the RVE by reducing its size for the same volume fraction.

Fig 8. shows also the comparison between the effective material properties of the numerical homogenization techniques and Mori-Tanaka approximations for the assumption that grains have been simulated like spherical particles. Furthermore, the results of Rule of Mixtures have been shown accompanied with the aforementioned comparison, but this analytical method does not take into consideration the shape of RVEs' inclusions. The above numerical and analytical results are in absolute compliance with the numerical results of the [10] which are named in the Fig.8 as "Reference Paper".

From these studies it can be concluded that for a given grain volume fraction, the results for elastic moduli showed no effect of the grain size. On the other hand, the numerical results gave a clear evidence of the Hall-Petch effect, which is a consequence of macroscopic strain being preferentially accumulated at grain boundaries.

\section{Conclusions}

The numerical homogenization tool has been developed for the prediction of the mechanical properties of Voronoi particles reinforced materials. Our numerical predictions were close to the results of Mori-Tanaka and Rule of Mixture approximations in the elastic case. Several investigations were made to determine the influence of the size of the Voronoi particles on the effective material properties. There were no significant variations with respect to the change in size of the particles.

It should be noticed that the model used in the present study accurately captures the properties of nanocrystalline metals. Specifically, there is clear evidence of the Hall-Petch effect, which is a result of macroscopic strain being preferentially accumulated at grain boundaries.

This grain boundary accumulation of strain is inherent to two-phase models, which assume a composite-like structure of nanometals. Based on this approach, such metals can be viewed 
as high volume fraction particulate systems; more precisely, as a system of variable strength particles joined by a thin boundary layer. The response of such a system to loads in the plastic strain regime cannot be estimated by a simple averaging of the response of its constituents because of strain partitioning.

Hence, an assumption of Hall-Petch law for the flow stress of grain interiors does not imply the Hall-Petch formula applies to the aggregate. In fact, in a future work, it will be demonstrated by the mere existence of critical grain size below which one can observe grain size softening.

In conclusion, it should be pointed out that the results of simulations conducted here demonstrate that the Hall-Petch relationship holds well for a two-phase model of nanocrystalline metals with variable-sized grains and they are validated utilizing experimental results.

The ICARUS project has received funding from the European Union's Horizon 2020 research and innovation programme under grant agreement No 713514.

\section{References}

1. K. Kumar, H. Van Swygenhoven, S. Suresh, Acta Mater. 51, 19 (2003).

2. M. Meyers, A. Mishra, D. Benson, Prog. Mater. Sci. 51, 4 (2006).

3. M. Kulczyk, W. Pachla, A. Mazur., R. Diduszko, H. Garbacz, M. Lewandowska, W. Łojkowski, K. Kurzydłowski, Mater. Sci., 23, 3 (2005)

4. H. Kim, C. Suryanarayana, S. Kim, B. Chun., Powder Metall. 41, 3 (1998).

5. J. Hu, S. Han, G. Sun, S. Sun, Z. Jiang, G. Wang, J. Lian, Mater. Sci. Eng., A 618, pp.621$628(2014)$

6. E. Wachowicz, T. Ossowski and A. Kiejna, A., (2010) Phys Rev B, 81, (2010)

7. ANSYS" Academic Associate, Release 12.1, Help System

8. W. Callister, Fundamentals of materials science and engineering,(2005)

9. M. Muzyk, PhD Thesis, Warsaw University of Technology (2010).

10. G. Stan, R.F. Cook, Nanotech. 19 (2008).

11. R. Dobosz, M. Lewandowska, K. Kurzydlowski, Scr. Mater. 67, 4 (2012).

12. J. Christoffersen, J. Mech. Phy. Solids 31, 1 (1983).

13. S. Cheng, E. Ma, Y. Wang, L. Kecskes, K. Youssef, C. Koch, Acta Mater. 53, 4, pp.15211533 (2005).

14. D. Polk, B. Giessen and F. Gardner Mater. Sci. Eng. 23, 2, pp. 309-316 (1976). 